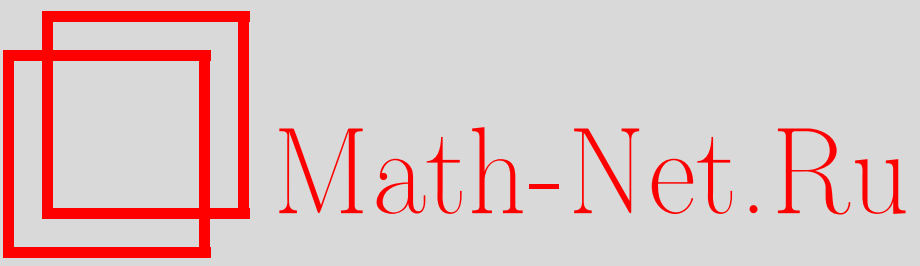

Г. Г. Ильюта, Разделенные разности для симметрических функций и альтернированные высшие порядки Брюа, УМН, 2001, том 56, выпуск 2, 217-218

DOI: https://doi.org/10.4213/rm390

Использование Общероссийского математического портала Math-Net.Ru подразумевает, что вы прочитали и согласны с пользовательским соглашением

http://www.mathnet.ru/rus/agreement

Параметры загрузки:

IP: 35.173 .219 .12

26 апреля 2023 г., 17:47:05 


\title{
РАЗДЕЛЕННЫЕ РАЗНОСТИ ДЛЯ СИММЕТРИЧЕСКИХ ФУНКЦИЙ И АЛЬТЕРНИРОВАННЫЕ ВЫСШИЕ ПОРЯДКИ БРЮА
}

\author{
Г. Г. ИльюТА
}

Элементы высших порядков Брюа [1] кодируют компоненты связности в пространстве невырожденных конфигураций точек из $\mathbb{R}^{2}[2]$. Дискриминантная гиперповерхность определяется с помощью интерполяции Лагранжа. В [3] интерполяционная формула Лагранжа обобщена для симметрических функций. Ниже для симметрических функций приводятся обобщенные формулы для разделенных разностей, подсказывающие определение обобщенных высших порядков Брюа (будем назьвать их альтернированными по причине их тесной связи с алштернированными ориентированньми матроидами), элементы которых кодируют компоненты связности соответствующего пространства конфигураций. Теорию ориентированных матроидов можно рассматривать как комбинаторную теорию детерминантных тождеств, и с этой точки зрения приведенное обобщение описьвается формулой для произведения максимальных миноров прямоугольной матрицы [4]. С точки зрения матроидов невырожденные конфигурации из [2] - это регулярные поднятия альтернированного ориентированного матроида [5; с. 62$]$. Над $\mathbb{C}$ приведенная конструкция приводит к обобщению дискриминантных конфигураций Манина-Шехтмана и к обобщенным группам кос (фундаментальная группа дополнения к дискриминантной конфигурации).

1. Обобщенная формула Лагранжа [3]. Пусть $f$ - симметрический полином от переменных $Y=\left\{y_{1}, \ldots, y_{r}\right\}$, степень по каждой из которых не превосходит $n-r$. Пространство $P_{n}$ всех таких полиномов имеет базис, состоящий из мономиальных симметрических полиномов $\varphi_{0, \ldots, 0}=1, \varphi_{1,0, \ldots, 0}=\sum y_{i}, \ldots, \varphi_{n-r, \ldots, n-r}=y_{1}^{n-r} \cdots y_{r}^{n-r}$. Через $C(n, r)$ обозначим множество $r$-элементных подмножеств в $\bar{n}=\{1, \ldots, n\}, I=\left\{i_{1}, \ldots, i_{r}\right\} \in C(n, r)$.

Пусть $X=\left\{x_{1}, \ldots, x_{n}\right\} \subset \mathbb{C}, x_{i} \neq x_{j}$ для $i \neq j$. Тогда

$$
f\left(y_{1}, \ldots, y_{r}\right)=\sum_{I \in C(n, r)} f\left(x_{i_{1}}, \ldots, x_{i_{r}}\right) \prod_{y \in Y, j \in \bar{n} \backslash I}\left(y-x_{j}\right) / \prod_{i \in I, j \in \bar{n} \backslash I}\left(x_{i}-x_{j}\right) .
$$

2. Формулы для разделенных разностей. Обозначения: $f(Y)$ - аналитическая симметрическая функция, $V_{1}(X)=\prod_{i>j}\left(x_{i}-x_{j}\right), F(Y)=-V_{1}^{2}(Y) f(Y)($ для $r=1, F=f)$, $\omega(x)=\left(x-x_{1}\right) \cdots\left(x-x_{n}\right), C_{i}-$ простой контур на комплексной прямой с координатой $y_{i}$, охватывающий $X$. Сопоставим функции $f$ интерполяционньй полином вида (1) и назовем разделенной разностью для $f$ по переменным $Y$ в точках из $X$ (обозначение: $\left.\Delta_{X}^{Y}(f)\right)$ коэфффициент при $\varphi_{n-r, \ldots, n-r}$ в этом полиноме. В формулах $(4),(5)$ ниже предполагается, что $X \subset \mathbb{R}$.

ПРЕДЛОЖЕНИЕ 1. Для любъх различных $x_{i_{1}}, \ldots, x_{i_{r+1}} \in X$

$$
\begin{aligned}
& \Delta_{X}^{Y}(f)=\left(\frac{1}{2 \pi i}\right)^{r} \frac{1}{r !} \int_{C_{1}} \cdots \int_{C_{r}} \frac{F\left(y_{1}, \ldots, y_{r}\right)}{\omega\left(y_{1}\right) \cdots \omega\left(y_{r}\right)} d y_{1} \cdots d y_{r}
\end{aligned}
$$

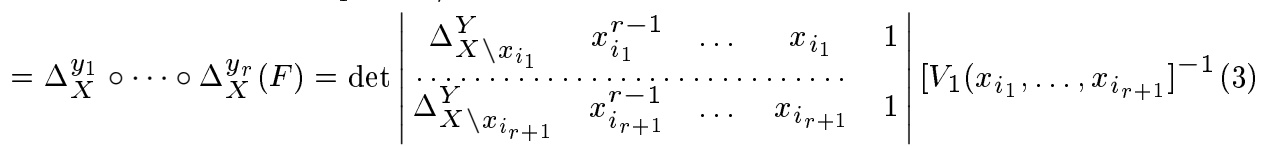

$$
\begin{aligned}
& =\int_{T_{1}} \cdots \int_{T_{r}} \frac{\partial^{r(n-1)} F}{\partial y_{1}^{n-1} \cdots \partial y_{r}^{n-1}}\left(t_{1}^{1} x_{1}+\cdots+t_{n}^{1} x_{n}, \ldots, t_{1}^{r} x_{1}+\cdots+t_{n}^{r} x_{n}\right) d t_{2}^{1} \cdots d t_{n}^{r} \\
& =\left.\frac{1}{((n-1) !)^{r}} \frac{\partial^{r(n-1)} F\left(y_{1}, \ldots, y_{r}\right)}{\partial y_{1}^{n-1} \ldots \partial y_{r}^{n-1}}\right|_{y_{1}=\xi_{1}, \ldots, y_{r}=\xi_{r}} .
\end{aligned}
$$

Здесь $T_{i}=\left\{\left(t_{1}^{i}, \ldots, t_{n}^{i}\right): \sum t_{j}^{i}=1, t_{j}^{i} \geqslant 0\right\}, \xi_{1}, \ldots, \xi_{r} \in\left[\min x_{i}, \max x_{i}\right]$.

Работа выполнена при поддержке Российского фонда фундаментальных исследований (грант № 98-01-00612) и NWO-RFBR 047.008.005. 
ЗАмечАния. 1) Поскольку вычет формы

$$
\Omega=\frac{F\left(y_{1}, \ldots, y_{r}\right)}{\omega\left(y_{1}\right) \cdots \omega\left(y_{r}\right)} d y_{1} \wedge \cdots \wedge d y_{r}
$$

в точке $\left(x_{i_{1}}, \ldots, x_{i_{r}}\right)$ равен $f\left(x_{i_{1}}, \ldots, x_{i_{r}}\right) / \prod\left(x_{i}-x_{j}\right), i \in I, j \in \bar{n} \backslash I$, если $x_{i_{j}}$ различны, и равен 0 , если среди них есть совпадаюшие, то сумма вычетов формы $\Omega / r$ ! во всех решениях системы $\omega\left(y_{1}\right)=\cdots=\omega\left(y_{r}\right)=0$ равна $\Delta_{X}^{Y}(f)$. Поэтому глобальная теорема о вычетах представляет $\Delta_{X}^{Y}(f)$ в виде интеграла от образа формы $\Omega / r$ ! при изоморффизме Долшбо [6; гл. 5].

2) Формула (2) позволяет определить $\Delta_{X}^{Y}$ для кратных узлов интерполяции.

3) Формула (3) представляет $\Delta_{X}^{Y}$ в виде композиции обычных разделенных разностей. Это позволяет обобщить и другие формулы, например, интерполяционную формулу Ньютона и формуль для разделенных разностей произведения и композиции функций [7].

4) В теории многообразий Шуберта и специальных функций большую роль играет оператор симметризации по транспозиции $\pi_{2}:\left(y_{1}, y_{2}\right) \mapsto\left(y_{2}, y_{1}\right)$

$$
\partial_{\pi_{2}} f\left(y_{1}, y_{2}\right)=\frac{f\left(y_{1}, y_{2}\right)-f\left(y_{2}, y_{1}\right)}{y_{2}-y_{1}} .
$$

Формула (3) в этом контексте отвечает симметризации по $(r+1)$-циклу $\pi_{r+1}:\left(y_{1}, \ldots, y_{r+1}\right) \mapsto$ $\left(y_{2}, \ldots, y_{r+1}, y_{1}\right)$.

3. Определитель Вандермонда для симметрических функций. Поиск коэффициентов при $\varphi_{m_{1}}, \ldots, m_{r}$ в интерполяционном полиноме можно рассматривать как решение линейной системы: подставляем все $\left(x_{i_{1}}, \ldots, x_{i_{r}}\right)$ в полином и получаем $C_{n}^{r}$ уравнений на коэффициенты. Обозначим через $V_{r}\left(x_{1}, \ldots, x_{n}\right)$ определитель этой системы.

ПРЕДЛОЖЕНИЕ 2.

$$
V_{r}\left(x_{1}, \ldots, x_{n}\right)=\left(V_{1}\left(x_{1}, \ldots, x_{n}\right)\right)^{C_{n-2}^{r-1}} .
$$

4. Альтернированные высшие порядки Брюа. Отображение $\varepsilon: C(m, n+1) \rightarrow$ $\{-1,1\}$ назовем $r$-допустимым, если для любого $p \in C(m, n+2)$ и любых $i_{1}, \ldots, i_{r+2} \in p$, $i_{1}<\cdots<i_{r+2},\left\{p \backslash i_{1}, p \backslash i_{3}, \ldots\right\} \neq \varepsilon^{-1}(1) \cap\left\{p \backslash i_{1}, p \backslash i_{2}, \ldots\right\} \neq\left\{p \backslash i_{2}, p \backslash i_{4}, \ldots\right\}$. Множество $r$-допустимых отображений назовем альтернированным высшим порядком Брюа $B(m, n, r)$ (частичньй порядок вводится аналогично [8]).

5. Пространство конфигураций. Набор $\mathrm{E}=\left\{\mathrm{E}_{I}=\left(y_{i_{1}, \ldots, i_{r}}, x_{i_{1}}, \ldots, x_{i_{r}}\right) \in \mathbb{R}^{r+1}\right.$, $I \in C(m, r), y_{i_{1}, \ldots, i_{r}}$ симметричны по индексам $\}$ назовем $n$-общим, если для любого $d \in$ $C(m, n+1)$ точки $\mathrm{E}_{I}, I \subset d$, не лежат на графике полинома из $P_{n+1}$ степени меньше $(n-r+1) r$, т.е. коэфффициент $\Delta_{d}^{Y}$ при $y_{1}^{n-r+1} \cdots y_{r}^{n-r+1}$ в соответствующем интерполяционном полиноме не равен 0. Определим $\varepsilon_{\mathrm{E}}: C(m, n+1) \rightarrow\{-1,1\}$ по формуле $\varepsilon_{\mathrm{E}}(d)=\operatorname{sign} \Delta_{d}^{Y}$.

ПреДЛоЖениЕ 3. Для любого $n$-общего набора Е отображение $\varepsilon_{\mathrm{E}}$-допустимо, т.е. определяет әлемент $B(m, n, r)$.

ЗАмечАниЕ. Из (3) следует, что векторы $\left(\Delta_{d \backslash i}^{Y}, x_{i}^{r-1}, \ldots, x_{i}, 1\right), i \in d$, определяют алштернированньй ориентированньй матроид.

\section{СПИСОК ЛИТЕРАТУРЫ}

[1] V. Manin, V. Schechtman // Adv. Stud. Pure Math. 1985. V. 44. P. 289-308. [2] Г. Г. Ильюта // Изв. РАН. Сер. матем. 1996. Т. 60. №6. С. 91-100. [3] W. Chen, J. Louck // Adv. Math. 1996. V. 117. P. 147-156. [4] B. Sturmfels, A. Zelevinsky // Adv. Math. 1993. V. 98. P. 65-112. [5] A. Bjørner, M. Las Vergnas, B. Sturmfels, N. White, G. M. Ziegler. Oriented Matroids. Cambridge: Cambridge Univ. Press, 1993. [6] Ф. Грифофитс, Д. Харрис. Принципы алгебраической геометрии. М.: Мир, 1982. [7] L. Verde-Star // Stud. Appl. Math. 1991. V. 85. P. 215-242. [8] G. Ziegler // Topology. 1993. V. 32. P. 259-279. 